\title{
The Role of Dementia Ward in an Acute Hospital
}

\section{Si Ching Lim*}

Department of Geriatric Medicine, Changi General Hospital, Singapore

${ }^{*}$ Corresponding author: Dr. Si Ching Lim, MB. ChB, MRCP (UK), Senior Consultant, Department of Geriatric Medicine, Changi General Hospital, Singapore, Tel: +65 68503362; E-mail: si_ching_lim@cgh.com.sg

Rec date: November 14, 2017; Acc date: November 24, 2017; Pub date: November 27, 2017

Copyright: $\odot 2017$ Lim SC. This is an open-access article distributed under the terms of the Creative Commons Attribution License, which permits unrestricted use, distribution, and reproduction in any medium, provided the original author and source are credited.

\begin{abstract}
Alzheimer disease International's data shows that there are 50 million people living with dementia worldwide in 2017 with someone in the world developing dementia every 3 seconds. The estimated global cost for dementia is about USD 1 trillion by 2018. In terms of healthcare utilization, the elderly with dementia are more likely to be hospitalized compared to their peers without dementia since dementia increases fall risk, injuries, infections and complications due to chronic diseases and drugs. During the hospital stay, the elderly patients with dementia are more likely to develop iatrogenic complications like delirium, falls and infections, with higher mortality is higher and longer length of stay.
\end{abstract}

Keywords: Dementia; Alzheimer disease; Behavioral symptoms

\section{Introduction}

Most of the elderly with dementia do not have a formal diagnosis especially among those residing in the developing countries. Without a formal diagnosis, the elderly misses out on treatment options, referrals to community services, education, support and counselling for the caregivers in terms of managing the declining cognition, emergence of behavioral symptoms and increasing care needs for their activities of daily living $[1,2]$.

\section{The Model of Care in Acute Hospitals}

In the acute hospital, the elderly with dementia frequently become agitated and restless because of unfamiliar environment and hospital staff. Furthermore, hospitalization upsets their daily routine. Incident delirium adds to the confusion and there may be communication barriers with the care staff, particularly if there is a language barrier. The agitated elderly patients are often restrained physically or pharmacologically to prevent falls or dislodgement of medical devices, particularly if they are restless and are unable to follow instructions. The use of restraints enforces immobility with undesirable consequences like delirium, agitation, falls, urinary infection, constipation, functional decline, etc. [2].

The traditional model of task oriented care in the acute hospital is not ideal for the elderly with dementia since their care needs are higher, with routine tasks and communication needing more time and patience. The presence of behavioral symptoms has been shown to cause considerable distress among the nursing staff [3]. Kitwood advocated for person centered care (PCC) in order to promote wellbeing and reduce emergence of challenging behavior [4]. However, the practice of person centered care in an acute hospital is challenging because of manpower and time constraints.

\section{Dementia ward in an acute general hospital}

This is a short introduction to a dementia ward with 20 beds in a teaching hospital in Singapore. The availability of dementia ward in a hospital is a niche in Singapore.

\section{Aims of dementia ward}

- Provide quality care and a calm environment for the elderly with delirium and dementia particularly those with behavioral symptoms.

- Minimize the use of physical and chemical restraints.

- Provide a platform for educating medical, nursing staffs and caregivers to care for elderly with challenging behaviors with nonpharmacological methods.

The model of care focuses on person centered care (PCC) rather than the traditional task oriented model, with a home like environment, individualized care plans and activities to minimize disorientation. Restraint use is strongly discouraged while freedom of movement within the ward is allowed in order to reduce functional decline while receiving medical care in an acute hospital (Figures 1-3).

The patients are either admitted directly from the emergency department or takeover from other disciplines. The patients have a diagnosis of delirium or dementia, often with challenging behaviors which are disruptive in the general wards. The use of physical and/or chemical restraints on patients is discouraged unless nonpharmacological means of behavioral management fails or if they are at risk of endangering themselves or others in the vicinity.

PCC involve individualized care plans and activities centered on the patients' likes and dislikes. In order to practice PCC, personal information on patient's daily routine, premorbid personality, food and drink preferences, sleep pattern, hobbies, previous occupations, family members, religious affiliations, events/persons which make the person happy/sad are obtained from the next of kin. Meaningful activities and care plans which include diet orders, personal grooming and bed times are set according to information collected. 
Page 2 of 3

The staffs have daily bedside teaching rounds and there is twice weekly support from the psychiatric liaison service. There is weekly multidisciplinary meeting to discuss rehab needs and discharge plans.

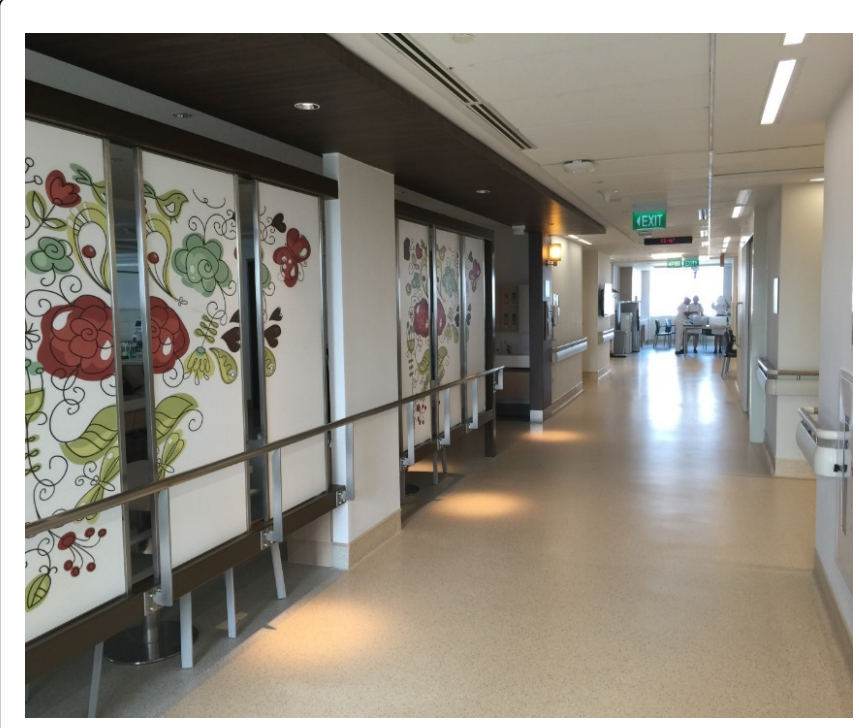

Figure 1: Corridor with gentle lighting and home like design, with grab bars to improve confidence while walking.

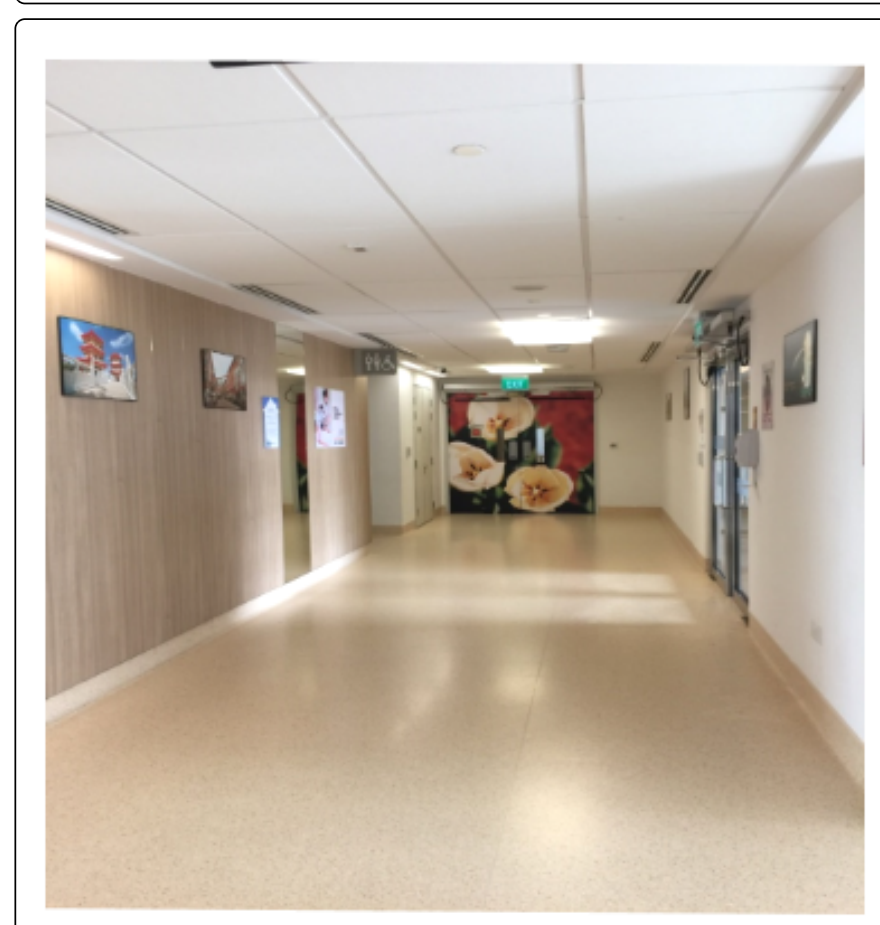

Figure 2: Main corridor with pictures on the wall for reminiscence while walking. Exit door disguised with picture to prevent the elderly wandering off.

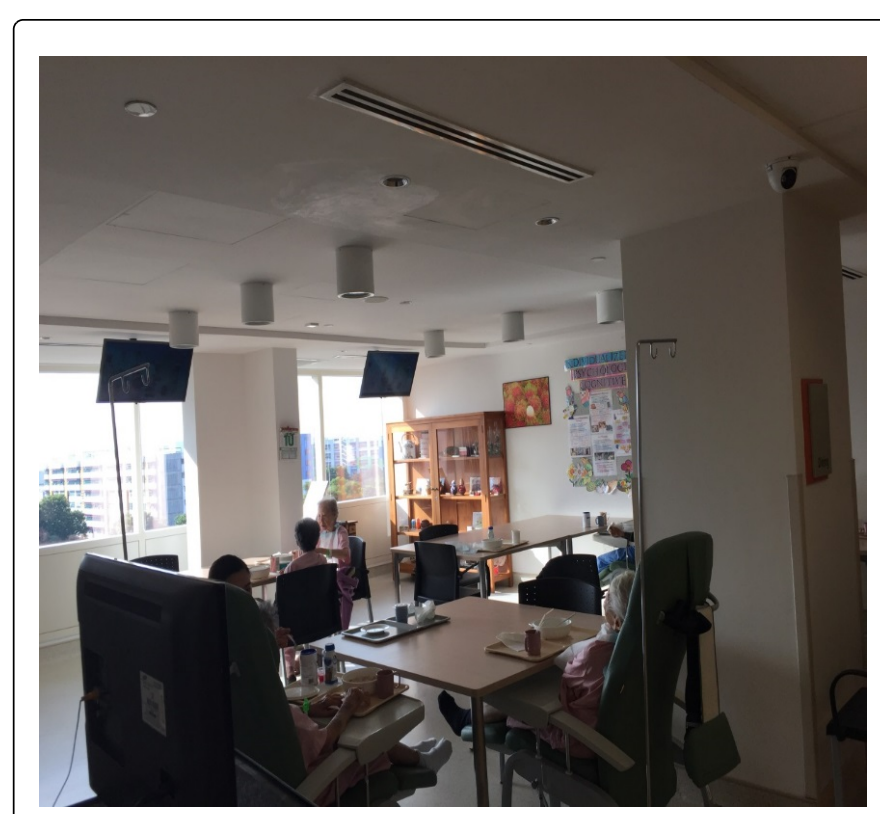

Figure 3: Communal dining areas with a cupboard of old artefacts for reminiscence therapy. Nursing staff providing assistance during meal time.

\section{Discussion}

\section{Outcome}

Data collection over 4 months on 80 patients showed $2 / 3$ of the patients exhibited difficult to manage behavioral symptoms. None of them were restrained physically in the dementia ward and $80 \%$ of the patients were discharged without any decline in their physical function. The family members receive individualized education and training on management of BPSD with $80 \%$ of the patients being successfully discharged back to their own home. There were 5 patients who were planned for nursing home initially but were eventually discharged home. Over $90 \%$ of the family members expressed satisfaction with the care model and thought the nursing staffs were well trained and passionate in their care for the elderly with dementia. The data was encouraging, even though the sample size was small, and the duration of study was short.

\section{Conclusion}

There is a role for a dementia ward in a busy general hospital to care for this special group of vulnerable elderly who might otherwise be physically restrained in the general ward with undesired consequences. The nursing staffs are compassionate and passionate in caring for this group of patients. In the longer term, the risk of burnout is high among the nurses because the workload is heavy and there is constant worry of patients falling or injuring themselves because of their high physical activities. The ongoing education, team spirit and support from the organization are essential to reduce burnout. 
Citation: Lim SC (2017) The Role of Dementia Ward in an Acute Hospital. J Gerontol Geriatr Res 6: 453. doi:10.4172/2167-7182.1000453

Page 3 of 3

\section{References}

1. World Alzheimer Report (2016) Improving healthcare for people living with dementia.

2. Lim SC (2016) Restraint use in the management of the elderly with dementia in hospital. Int Med Res Open J 1:1-4.
3. Hessler JB (2017) Behavioral and psychological symptoms in general hospital patients with dementia, distress for nurses and complications in care: Results of the general hospital study. Epidemiol Psychiatr Sci.

4. Kitwood T (1997) Dementia reconsidered: The person comes first. Open University Press, Buckingham, UK. 